\title{
Demiclosed principle and convergence theorems for asymptotically strictly pseudononspreading mappings and mixed equilibrium problems
}

Zhaoli Ma' and Lin Wang ${ }^{2 *}$

"Correspondence:

WL64mail@aliyun.com

${ }^{2}$ College of Statistics and

Mathematics, Yunnan University of Finance and Economics, Long quan

Road, Kunming, 650221, China

Full list of author information is

available at the end of the article

\begin{abstract}
In this paper, the demiclosed principle for a k-asymptotically strictly pseudononspreading mapping is shown. Meanwhile, an iterative scheme is introduced to approximate a common element of the set of common fixed points of $k$-asymptotically strictly pseudononspreading mappings and the set of solutions of mixed equilibrium problems in Hilbert spaces, and some weak and strong convergence theorems are proved. The results presented in this paper improve and extend some recent corresponding results.
\end{abstract}

MSC: 47H09; 47J25

Keywords: k-asymptotically strictly pseudononspreading mapping; mixed equilibrium problem; weak and strong convergence; demiclosed principle

\section{Introduction}

Let $H$ be a real Hilbert space with the inner product $\langle\cdot, \cdot\rangle$ and the norm $\|\cdot\|$. Let $C$ be a nonempty closed convex subset of $H$ and $F: C \times C \rightarrow R$ be a bifunction, where $R$ is the set of real numbers. The equilibrium problem (for short, $E P$ ) is to find $x^{*} \in C$ such that

$$
F\left(x^{*}, y\right) \geq 0, \quad \forall y \in C .
$$

The set of solutions of $E P$ is denoted by $E P(F)$. Given a mapping $T: C \rightarrow C$, let $F(x, y)=$ $\langle T x, y-x\rangle$ for all $x, y \in C$. Then $x^{*} \in E P(F)$ if and only if $x^{*} \in C$ is a solution of the variational inequality $\langle T x, y-x\rangle \geq 0$ for all $y \in C$, i.e., $x^{*}$ is a solution of the variational inequality.

Let $\varphi: C \rightarrow R \cup\{+\infty\}$ be a function. The mixed equilibrium problem (for short, MEP) is to find $x^{*} \in C$ such that

$$
F\left(x^{*}, y\right)+\varphi(y)-\varphi\left(x^{*}\right) \geq 0, \quad \forall y \in C .
$$

The set of solutions of $M E P$ is denoted by $\operatorname{MEP}(F, \varphi)$.

If $\varphi=0$, then mixed equilibrium problem (1.2) reduces to (1.1).

(2014 Ma and Wang: licensee Springer. This is an Open Access article distributed under the terms of the Creative Commons Attribution License (http://creativecommons.org/licenses/by/2.0), which permits unrestricted use, distribution, and reproduction in any medium, provided the original work is properly cited. 
If $F=0$, then mixed equilibrium problem (1.2) reduces to the following convex minimization problem:

$$
\text { Find } x^{*} \in C \text { such that } \varphi(y) \geq \varphi\left(x^{*}\right), \quad \forall y \in C \text {. }
$$

The set of solutions of (1.3) is denoted by $\operatorname{CMP}(\varphi)$.

The mixed equilibrium problem (MEP) includes several important problems arising in physics, engineering, science optimization, economics, transportation, network and structural analysis, Nash equilibrium problems in noncooperative games and others. It has been shown that variational inequalities and mathematical programming problems can be viewed as a special realization of abstract equilibrium problems (e.g., [1, 2]). Many authors have proposed some useful methods to solve the $E P, M E P$; see, for instance, [1-8] and the references therein.

Let $H$ be a real Hilbert space and $C$ be a nonempty closed convex subset of $H$. Following Kohsaka and Takahashi [9-11], a mapping $T: C \rightarrow C$ is said to be nonspreading if

$$
2\|T x-T y\|^{2} \leq\|T x-y\|^{2}+\|T y-x\|^{2} \quad \text { for all } x, y \in C .
$$

It is easy to see that the above inequality is equivalent to

$$
\|T x-T y\|^{2} \leq\|x-y\|^{2}+2\langle x-T x, y-T y\rangle \quad \text { for all } x, y \in C .
$$

In 1967, Browder and Petryshyn [12] introduced the concept of $k$-strictly pseudononspreading mapping.

Definition 1.1 [12] Let $H$ be a real Hilbert space. A mapping $T: D(T) \subset H \rightarrow H$ is said to be $k$-strictly pseudononspreading if there exists $k \in[0,1)$ such that

$$
\|T x-T y\|^{2} \leq\|x-y\|^{2}+k\|x-T x-(y-T y)\|^{2}+2\langle x-T x, y-T y\rangle, \quad \forall x, y \in D(T) .
$$

Clearly, every nonspreading mapping is $k$-strictly pseudononspreading.

In 2012, Osilike [13] introduced a class of nonspreading type mappings, which is more general than the mappings studied in [14] in Hilbert spaces, and proved some weak and strong convergence theorems in real Hilbert spaces. Recently, Chang [15] studied the multiple-set split feasibility problem for asymptotically strict pseudocontraction in the framework of infinite-dimensional Hilbert spaces.

Definition 1.2 [15] Let $H$ be a real Hilbert space. A mapping $T: D(T) \subset H \rightarrow H$ is said to be a $k$-asymptotically strict pseudocontraction if there exist a constant $k \in[0,1)$ and a sequence $\left\{k_{n}\right\} \subset[1, \infty)$ with $k_{n} \rightarrow 1(n \rightarrow \infty)$ such that

$$
\left\|T^{n} x-T^{n} y\right\|^{2} \leq k_{n}\|x-y\|^{2}+k\left\|x-T^{n} x-\left(y-T^{n} y\right)\right\|^{2}
$$

holds for all $x, y \in D(T)$. 
Definition 1.3 Let $C$ be a nonempty subset of a real Hilbert space $H$. A mapping $T$ : $C \rightarrow C$ is said to be $k$-asymptotically strictly pseudononspreading if there exist a constant $k \in[0,1)$ and a sequence $\left\{k_{n}\right\} \subset[1, \infty)$ with $k_{n} \rightarrow 1(n \rightarrow \infty)$ such that

$$
\begin{aligned}
& \left\|T^{n} x-T^{n} y\right\|^{2} \leq k_{n}\|x-y\|^{2}+k\left\|x-T^{n} x-\left(y-T^{n} y\right)\right\|^{2}+2\left\langle x-T^{n} x, y-T^{n} y\right\rangle, \\
& \quad \forall x, y \in C .
\end{aligned}
$$

It is easy to see that the class of $k$-asymptotically strictly pseudononspreading mappings is more general than the classes of $k$-strictly pseudononspreading mappings and $k$-asymptotically strict pseudocontractions.

Example 1.4 Let $X=l^{2}$ with the norm $\|\cdot\|$ defined by

$$
\|x\|=\sqrt{\sum_{i=1}^{\infty} x_{i}^{2}}, \quad \forall x=\left(x_{1}, x_{2}, \ldots, x_{n}, \ldots\right) \in X,
$$

and $C=\left\{x=\left(x_{1}, x_{2}, \ldots, x_{n}, \ldots\right) \mid x_{i} \in R^{1}, i=1,2, \ldots\right\}$ be an orthogonal subspace of $X$ (i.e., $\forall x, y \in C$, we have $\langle x, y\rangle=0)$. It is obvious that $C$ is a nonempty closed convex subset of $X$. For each $x=\left(x_{1}, x_{2}, \ldots, x_{n}, \ldots\right) \in C$, we define the mapping $T: C \rightarrow C$ by

$$
T x= \begin{cases}\left(x_{1}, x_{2}, \ldots, x_{n}, \ldots\right) & \text { if } \prod_{i=1}^{\infty} x_{i}<0 \\ \left(-x_{1},-x_{2}, \ldots,-x_{n}, \ldots\right) & \text { if } \prod_{i=1}^{\infty} x_{i} \geq 0\end{cases}
$$

Next we prove that $T$ is a $k$-asymptotically strictly pseudononspreading mapping.

In fact, for any $x, y \in C$.

Case 1. If $\prod_{i=1}^{\infty} x_{i}<0$ and $\prod_{i=1}^{\infty} y_{i}<0$, then we have $T^{n} x=x, T^{n} y=y$, and so inequality (1.4) holds for any $k \in[0,1)$.

Case 2. If $\prod_{i=1}^{\infty} x_{i}<0$ and $\prod_{i=1}^{\infty} y_{i} \geq 0$, then we have that $T^{n} x=x, T^{n} y=(-1)^{n} y$. This implies that

$$
\left\{\begin{array}{l}
\left\|T^{n} x-T^{n} y\right\|^{2}=\left\|x-(-1)^{n} y\right\|^{2}=\|x\|^{2}+\|y\|^{2} \\
k_{n}\|x-y\|^{2}=k_{n}\left(\|x\|^{2}+\|y\|^{2}\right) ; \\
\left\|x-T^{n} x-\left(y-T^{n} y\right)\right\|^{2}=\left[1-(-1)^{n}\right]^{2}\|y\|^{2} \\
2\left\langle x-T^{n} x, y-T^{n} y\right\rangle=0 .
\end{array}\right.
$$

Therefore inequality (1.4) holds for any $k \in[0,1)$.

Case 3. If $\prod_{i=1}^{\infty} x_{i} \geq 0$ and $\prod_{i=1}^{\infty} y_{i}<0$, then we have that $T^{n} x=(-1)^{n} x, T^{n} y=y$. Therefore we obtain

$$
\left\{\begin{array}{l}
\left\|T^{n} x-T^{n} y\right\|^{2}=\left\|(-1)^{n} x-y\right\|^{2}=\|x\|^{2}+\|y\|^{2} \\
k_{n}\|x-y\|^{2}=k_{n}\left(\|x\|^{2}+\|y\|^{2}\right) \\
\left\|x-T^{n} x-\left(y-T^{n} y\right)\right\|^{2}=\left[1-(-1)^{n}\right]^{2}\|x\|^{2} \\
2\left\langle x-T^{n} x, y-T^{n} y\right\rangle=0 .
\end{array}\right.
$$

So, inequality (1.4) holds for any $k \in[0,1)$. 
Case 4. If $\prod_{i=1}^{\infty} x_{i} \geq 0$ and $\prod_{i=1}^{\infty} y_{i} \geq 0$, then we have $T^{n} x=(-1)^{n} x, T^{n} y=(-1)^{n} y$. Hence we have

$$
\left\{\begin{array}{l}
\left\|T^{n} x-T^{n} y\right\|^{2}=\left\|(-1)^{n} x-(-1)^{n} y\right\|^{2}=\|x-y\|^{2}=\|x\|^{2}+\|y\|^{2} \\
k_{n}\|x-y\|^{2}=k_{n}\left(\|x\|^{2}+\|y\|^{2}\right) \\
\left\|x-T^{n} x-\left(y-T^{n} y\right)\right\|^{2}=\left[1-(-1)^{n}\right]^{2}\|x-y\|^{2}=\left[1-(-1)^{n}\right]^{2}\left(\|x\|^{2}+\|y\|^{2}\right) \\
2\left\langle x-T^{n} x, y-T^{n} y\right\rangle=0 .
\end{array}\right.
$$

Thus inequality (1.4) still holds for any $k \in[0,1)$. Therefore the mapping defined by (1.5) is a $k$-asymptotically strictly pseudononspreading mapping.

A mapping $T: C \rightarrow C$ is said to be uniformly $L$-Lipschitzian if there exists a constant $L>0$ such that for all $(x, y) \in H \times H$,

$$
\left\|T^{n} x-T^{n} y\right\| \leq L\|x-y\|
$$

A Banach space $E$ is said to satisfy Opial's condition if, for any sequence $\left\{x_{n}\right\}$ in $E, x_{n} \rightarrow x$ implies that $\lim \sup _{n \rightarrow \infty}\left\|x_{n}-x\right\|<\lim \sup _{n \rightarrow \infty}\left\|x_{n}-y\right\|$ for all $y \in E$ with $y \neq x$. It is well known that every Hilbert space satisfies Opial's condition.

A mapping $T$ with domain $D(T)$ and range $R(T)$ in $E$ is said to be demiclosed at $p$ if whenever $\left\{x_{n}\right\}$ is a sequence in $D(T)$ such that $\left\{x_{n}\right\}$ converges weakly to $x^{*} \in D(T)$ and $\left\{T x_{n}\right\}$ converges strongly to $p$, then $T x^{*}=p$.

$T$ is said to be semi-compact if for any bounded sequence $\left\{x_{n}\right\} \subset H$ with $\lim _{n \rightarrow \infty} \| x_{n}-$ $T x_{n} \|=0$, there exists a subsequence $\left\{x_{n_{i}}\right\}$ of $\left\{x_{n}\right\}$ such that $\left\{x_{n_{i}}\right\}$ converges strongly to a point $x^{*} \in H$.

Recently, Zhao and Chang [16] proposed the following algorithm for solving $k$-strictly pseudononspreading mappings and equilibrium problem in Hilbert spaces.

$$
\left\{\begin{array}{l}
F\left(u_{n}, y\right)+\frac{1}{r_{n}}\left\langle y-u_{n}, u_{n}-x_{n}\right\rangle \geq 0, \quad \forall y \in C, \\
x_{n+1}=\alpha_{0, n} u_{n}+\sum_{i=1}^{\infty} \alpha_{i, n} S_{i, \beta} u_{n}
\end{array}\right.
$$

where $S_{i, \beta}:=\beta I+(1-\beta) S_{i}, \alpha_{i, n} \subset(0,1)$. Under some suitable conditions, they proved that the sequences $\left\{x_{n}\right\},\left\{y_{n}\right\}$ weakly and strongly converge to a solution of the problem $x^{*} \in$ $\bigcap_{i=1}^{\infty} F\left(S_{i}\right) \cap E P(F)$.

For finding a split feasibility problem for $k$-strictly pseudononspreading mappings in a Hilbert space, in [17], Quan and Chang presented the following iterative method:

$$
\left\{\begin{array}{l}
x_{1} \in H_{1} \text { chosen arbitrarily, } \\
u_{n}=x_{n}+\gamma A^{*}\left(T_{n(\bmod N)}-I\right) A x_{n}, \\
x_{n+1}=\left(1-\alpha_{n}\right) u_{n}+\alpha_{n} S_{n(\bmod N)} u_{n},
\end{array}\right.
$$

where $\gamma$ is a constant and $\gamma \in\left(0, \frac{1-\kappa}{\lambda}\right), \lambda$ is the spectral of the operator $A^{*} A, \kappa=$ $\max \left\{\kappa_{1}, \kappa_{2}, \ldots, \kappa_{N}\right\}$, and $\left\{\alpha_{n}\right\}$ is a sequence in $(0,1-\varrho]$ with $\varrho=\max \left\{\varrho_{1}, \varrho_{2}, \ldots, \varrho_{N}\right\}$. Under some suitable conditions, they proved that $\left\{x_{n}\right\}$ weakly and strongly converges to a split fixed point $x^{*} \in \Gamma$.

Inspired and motivated by the recent works of Zhao and Chang [16], Quan and Chang [17], etc., in this paper, we propose an iterative scheme to approximate a common element of the set of solutions of $k$-asymptotically strictly pseudononspreading mappings 
and mixed equilibrium problem in infinite-dimensional Hilbert spaces. Some weak and strong convergence theorems are proved. At the same time, the demiclosed principle of a $k$-asymptotically strictly pseudononspreading mapping is shown. The results presented in this paper improve and extend some recent corresponding results.

\section{Preliminaries}

Throughout this paper, we denote the strong convergence and weak convergence of a sequence $\left\{x_{n}\right\}$ to a point $x \in X$ by $x_{n} \rightarrow x, x_{n} \rightarrow x$, respectively.

Let $H$ be a Hilbert space with the inner product $\langle\cdot, \cdot\rangle$ and the norm $\|\cdot\|$, let $C$ be a nonempty closed convex subset of $H$. For every point $x \in H$, there exists a unique nearest point of $C$, denoted by $P_{C} x$, such that $\left\|x-P_{C} x\right\| \leq\|x-y\|$ for all $y \in C$. Such a $P_{C}$ is called the metric projection from $H$ onto $C$. It is well known that $P_{C}$ is a firmly nonexpansive mapping from $H$ to $C$, i.e.,

$$
\left\|P_{C} x-P_{C} y\right\|^{2} \leq\left\langle P_{C} x-P_{C} y, x-y\right\rangle, \quad \forall x, y \in H .
$$

Further, for any $x \in H$ and $z \in C, z=P_{C} x$ if and only if

$$
\langle x-z, z-y\rangle \geq 0, \quad \forall y \in C
$$

For solving mixed equilibrium problems, we assume that the bifunction $F: C \times C \rightarrow R$ satisfies the following conditions:

(A1) $F(x, x)=0, \forall x \in C$;

(A2) $F(x, y)+F(y, x) \leq 0, \forall x, y \in C$;

(A3) For all $x, y, z \in C, \lim _{t \downarrow 0} F(t z+(1-t) x, y) \leq F(x, y)$;

(A4) For each $x \in C$, the function $y \longmapsto F(x, y)$ is convex and lower semi-continuous.

Lemma 2.1 [18] Let $C$ be a nonempty closed convex subset of a Hilbert space H. Let $F$ be a bifunction from $C \times C$ to $R$ satisfying (A1)-(A4), and let $\varphi: C \rightarrow R \cup\{+\infty\}$ be a proper lower semi-continuous and convex function such that $C \cap \operatorname{dom} \varphi \neq \emptyset$. For $r>0$ and $x \in C$, define a mapping $T_{r}: H \rightarrow C$ as follows:

$$
T_{r}(x)=\left\{z \in C: F(z, y)+\varphi(y)-\varphi(z)+\frac{1}{r}\langle y-z, z-x\rangle \geq 0, \forall y \in C\right\}, \quad \forall x \in H .
$$

Then

(1) For each $x \in H, T_{r}(x) \neq \emptyset$;

(2) $T_{r}$ is single-valued;

(3) $T_{r}$ is firmly nonexpansive, that is, $\forall x, y \in H$,

$$
\left\|T_{r} x-T_{r} y\right\|^{2} \leq\left\langle T_{r} x-T_{r} y, x-y\right\rangle
$$

(4) $F\left(T_{r}\right)=M E P(F, \varphi)$;

(5) $\operatorname{MEP}(F, \varphi)$ is closed and convex.

Lemma 2.2 [13] Let $H$ be a real Hilbert space. Then the following results hold: 
(i) For all $x, y \in H$ and for all $t \in[0,1]$,

$$
\|t x+(1-t) y\|^{2}=t\|x\|^{2}+(1-t)\|y\|^{2}-t(1-t)\|x-y\|^{2} .
$$

(ii) $\|x+y\|^{2} \leq\|x\|^{2}+2\langle y, x+y\rangle$.

(iii) If $\left\{x_{n}\right\}_{n=1}^{\infty}$ is a sequence in $H$ which converges weakly to $z \in H$, then

$$
\limsup _{n \rightarrow \infty}\left\|x_{n}-y\right\|^{2}=\limsup _{n \rightarrow \infty}\left\|x_{n}-z\right\|^{2}+\|z-y\|^{2}, \quad \forall y \in H .
$$

The demiclosed principle and the closeness and convexity of the set of fixed points of a nonlinear mapping play very important roles in investigating many nonlinear problems. We now show the demiclosed principle of $k$-asymptotically strictly pseudononspreading mapping and the closeness and convexity of the set of fixed points of such a mapping, respectively.

Lemma 2.3 Let $C$ be a nonempty closed convex subset of a real Hilbert space $H$ and let $T: C \rightarrow C$ be a continuous $k$-asymptotically strictly pseudononspreading mapping. If $F(T) \neq \emptyset$, then it is closed and convex.

Proof Let $\left\{x_{n}\right\}_{n=1}^{\infty} \subset F(T)$ be a sequence which converges to $x \in C$, we show that $x \in F(T)$.

$$
\left\|T^{n} x-x\right\|=\left\|T^{n} x-x_{n}+x_{n}-x\right\| \leq\left\|T^{n} x-T^{n} x_{n}\right\|+\left\|x_{n}-x\right\| .
$$

Since $T$ is $k$-asymptotically strictly pseudononspreading, we have

$$
\begin{aligned}
\left\|T^{n} x-T^{n} x_{n}\right\|^{2} \leq & k_{n}\left\|x-x_{n}\right\|^{2}+k\left\|\left(T^{n} x_{n}-x_{n}\right)-\left(T^{n} x-x\right)\right\|^{2} \\
& +2\left\langle x-T^{n} x, x_{n}-T^{n} x_{n}\right\rangle \\
= & k_{n}\left\|x_{n}-x\right\|^{2}+k\left\|T^{n} x-x\right\|^{2} \\
\leq & \left(\sqrt{k_{n}}\left\|x_{n}-x\right\|+\sqrt{k}\left\|T^{n} x-x\right\|\right)^{2} .
\end{aligned}
$$

Using (2.4) in (2.3), we obtain

$$
\left\|T^{n} x-x\right\| \leq \sqrt{k_{n}}\left\|x_{n}-x\right\|+\sqrt{k}\left\|x-T^{n} x\right\|+\left\|x_{n}-x\right\|,
$$

so

$$
\begin{aligned}
& (1-\sqrt{k})\left\|T^{n} x-x\right\| \leq\left(\sqrt{k_{n}}+1\right)\left\|x_{n}-x\right\|, \\
& \left\|T^{n} x-x\right\| \leq \frac{\sqrt{k_{n}+1}}{1-\sqrt{k}}\left\|x_{n}-x\right\| .
\end{aligned}
$$

Since $k_{n} \rightarrow 1$ and $\left\|x_{n}-x\right\| \rightarrow 0$ as $n \rightarrow \infty$, we get that $\lim _{n \rightarrow \infty}\left\|T^{n} x-x\right\|=0$. Since $T$ is continuous, which implies that $x=\lim _{n \rightarrow \infty} T^{n} x=\lim _{n \rightarrow \infty} T\left(T^{n-1} x\right)=T\left(\lim _{n \rightarrow \infty} T^{n-1} x\right)=$ $T x$. Hence, $x \in F(T)$.

Now, we show that $F(T)$ is convex. 
For $x, y \in F(T)$ and $t \in(0,1)$, put $z=t x+(1-t) y$. We show that $z=T z$. In fact, we have

$$
\begin{aligned}
\left\|z-T^{n} z\right\|^{2}= & \|z\|^{2}-2\left\langle z, T^{n} z\right\rangle+\left\|T^{n} z\right\|^{2} \\
= & \|z\|^{2}-2\left\langle t x+(1-t) y, T^{n} z\right\rangle+\left\|T^{n} z\right\|^{2} \\
= & \|z\|^{2}-2 t\left\langle x, T^{n} z\right\rangle-2(1-t)\left\langle y, T^{n} z\right\rangle+\left\|T^{n} z\right\|^{2} \\
= & \|z\|^{2}+t\|x\|^{2}-2 t\left\langle x, T^{n} z\right\rangle+t\left\|T^{n} z\right\|^{2}+(1-t)\|y\|^{2} \\
& -2(1-t)\left\langle y, T^{n} z\right\rangle+(1-t)\left\|T^{n} z\right\|^{2}-t\|x\|^{2}-(1-t)\|y\|^{2} \\
= & \|z\|^{2}+t\left\|x-T^{n} z\right\|^{2}+(1-t)\left\|y-T^{n} z\right\|^{2}-t\|x\|^{2}-(1-t)\|y\|^{2} \\
\leq & \|z\|^{2}+t\left[k_{n}\|x-z\|^{2}+k\left\|x-T^{n} x-\left(z-T^{n} z\right)\right\|^{2}+2\left\langle x-T^{n} x, z-T^{n} z\right\rangle\right] \\
& +(1-t)\left[k_{n}\|y-z\|^{2}+k\left\|y-T^{n} y-\left(z-T^{n} z\right)\right\|^{2}\right. \\
& \left.+2\left\langle y-T^{n} y, z-T^{n} z\right\rangle\right]-t\|x\|^{2}-(1-t)\|y\|^{2} \\
\leq & \|z\|^{2}+t k_{n}\|x-z\|^{2}+t k\left\|z-T^{n} z\right\|^{2}+(1-t) k_{n}\|y-z\|^{2} \\
& +(1-t) k\left\|z-T^{n} z\right\|^{2}-t\|x\|^{2}-(1-t)\|y\|^{2} \\
= & \|z\|^{2}+t k_{n}\|x-z\|^{2}+(1-t) k_{n}\|y-z\|^{2} \\
& +k\left\|z-T^{n} z\right\|^{2}-t\|x\|^{2}-(1-t)\|y\|^{2} .
\end{aligned}
$$

So,

$$
\begin{aligned}
(1-k)\left\|z-T^{n} z\right\|^{2} \leq & \|z\|^{2}+t k_{n}\langle x-z, x-z\rangle \\
& +(1-t) k_{n}\langle y-z, y-z\rangle-t\|x\|^{2}-(1-t)\|y\|^{2} \\
\leq & \left(k_{n}-1\right)\left[t\|x\|^{2}+(1-t)\|y\|^{2}+\|z\|^{2}\right] .
\end{aligned}
$$

Since $k_{n} \rightarrow 1$ as $n \rightarrow \infty$, we obtain that $\lim _{n \rightarrow \infty}\left\|z-T^{n} z\right\|^{2}=0$, which implies that $\lim _{n \rightarrow \infty} T^{n} z=z, z=\lim _{n \rightarrow \infty} T^{n} z=T \lim _{n \rightarrow \infty}\left(T^{n-1} z\right)=T z$. Hence, $z \in F(T)$, which means that $F(T)$ is convex.

Lemma 2.4 Let $C$ be a nonempty closed convex subset of a real Hilbert space $H$, and let $T$ : $C \rightarrow C$ be a k-asymptotically strictly pseudononspreading and uniformly L-Lipschitzian mapping. Then, for any sequence $\left\{x_{n}\right\}$ in $C$ converging weakly to a point $p$ and $\left\{\left\|x_{n}-T x_{n}\right\|\right\}$ converging strongly to 0 , we have $p=T p$.

Proof Since $\lim _{n \rightarrow \infty}\left\|x_{n}-T x_{n}\right\|=0$, by induction we can prove that

$$
\lim _{n \rightarrow \infty}\left\|x_{n}-T^{m} x_{n}\right\|=0 \quad \text { for each } m \geq 1
$$

In fact, it is obvious that the conclusion is true for $m=1$. Suppose that the conclusion holds for $m>1$, now we prove that the conclusion is also true for $m+1$.

Indeed, since $T$ is uniformly $L$-Lipschitzian, we have

$$
\left\|x_{n}-T^{m+1} x_{n}\right\| \leq\left\|x_{n}-T^{m} x_{n}\right\|+\left\|T^{m} x_{n}-T^{m+1} x_{n}\right\| \leq\left\|x_{n}-T^{m} x_{n}\right\|+L\left\|x_{n}-T x_{n}\right\| .
$$

So, $\lim _{n \rightarrow \infty}\left\|x_{n}-T^{m+1} x_{n}\right\|=0$. 
For each $x \in H$, define $f: H \rightarrow[0, \infty)$ by

$$
f(x):=\limsup _{n \rightarrow \infty}\left\|x_{n}-x\right\|^{2}
$$

Then from Lemma 2.2 we have

$$
f(x)=\limsup _{n \rightarrow \infty}\left\|x_{n}-p\right\|^{2}+\|p-x\|^{2}, \quad \forall x \in H .
$$

Thus, for any $x \in H, f(x)=f(p)+\|p-x\|^{2}$ and

$$
\begin{aligned}
f\left(T^{m} p\right)= & f(p)+\left\|p-T^{m} p\right\|^{2} \\
f\left(T^{m} p\right)= & \limsup _{n \rightarrow \infty}\left\|x_{n}-T^{m} p\right\|^{2} \\
= & \limsup _{n \rightarrow \infty}\left\|x_{n}-T^{m} x_{n}+T^{m} x_{n}-T^{m} p\right\|^{2} \\
= & \limsup _{n \rightarrow \infty}\left\|T^{m} x_{n}-T^{m} p\right\|^{2} \\
\leq & \limsup _{n \rightarrow \infty}\left[k_{m}\left\|x_{n}-p\right\|^{2}+k\left\|x_{n}-T^{m} x_{n}-\left(p-T^{m} p\right)\right\|^{2}\right. \\
& \left.+2\left\langle x_{n}-T^{m} x_{n}, p-T^{m} p\right\rangle\right] \\
= & \limsup _{n \rightarrow \infty} k_{m}\left\|x_{n}-p\right\|^{2}+k\left\|p-T^{m} p\right\|^{2} \\
= & k_{m} f(p)+k\left\|p-T^{m} p\right\|^{2} .
\end{aligned}
$$

It follows from (2.7) and (2.8) that

$$
(1-k)\left\|p-T^{m} p\right\| \leq\left(k_{m}-1\right)\|f(p)\| .
$$

That is,

$$
\lim _{m \rightarrow \infty}\left\|p-T^{m} p\right\|=0
$$

Hence we have

$$
\begin{aligned}
\|T p-p\| & \leq\left\|T p-T^{m} p\right\|+\left\|T^{m} p-p\right\| \\
& \leq L\left\|p-T^{m-1} p\right\|+\left\|T^{m} p-p\right\| .
\end{aligned}
$$

This is $p=T p$, as desired. The proof is completed.

Lemma 2.5 [19] Let the number sequences $\left\{a_{n}\right\}$ and $\left\{\alpha_{n}\right\}$ satisfy

$$
a_{n+1} \leq\left(1+\alpha_{n}\right) a_{n}, \quad \forall n \geq 1,
$$

where $a_{n} \geq 0, \alpha_{n} \geq 0$ and $\sum_{n=1}^{\infty} \alpha_{n}<\infty$. Then

(1) $\lim _{n \rightarrow \infty} a_{n}$ exists;

(2) if $\liminf _{n \rightarrow \infty} a_{n}=0$, then $\lim _{n \rightarrow \infty} a_{n}=0$. 


\section{Main results}

Theorem 3.1 Let $C$ be a nonempty and closed convex subset of a real Hilbert space $H$, let $F$ be a bifunction from $C \times C$ to $R$ satisfying (A1)-(A4), and let $\varphi: C \rightarrow R \cup\{+\infty\}$ be a proper lower semi-continuous and convex function such that $C \cap \operatorname{dom} \varphi \neq \emptyset$. Let $T_{i}$ : $C \rightarrow C$ be a uniformly $L_{i}$-Lipschitzian and $\tau_{i}$-asymptotically strictly pseudononspreading mapping with the sequence $\left\{k_{n}\right\} \subset[1,+\infty)$ such that $\sum_{n=1}^{\infty}\left(k_{n}-1\right)<\infty$, let $S_{i}: C \rightarrow C$ be a uniformly $\widetilde{L}_{i}$-Lipschitzian and $l_{i}$-asymptotically strictly pseudononspreading mapping with the sequence $\left\{\rho_{n}\right\} \subset[1,+\infty)$ such that $\sum_{n=1}^{\infty}\left(\rho_{n}-1\right)<\infty, i=1,2, \ldots, N$. Let $\left\{x_{n}\right\}$ be a sequence generated by

$$
\left\{\begin{array}{l}
\forall x_{1} \in C, \\
F\left(u_{n}, y\right)+\varphi(y)-\varphi\left(u_{n}\right)+\frac{1}{r_{n}}\left\langle y-u_{n}, u_{n}-x_{n}\right\rangle \geq 0, \quad \forall y \in C, \\
y_{n}=\left(1-\beta_{n}\right) u_{n}+\beta_{n} T_{n(\bmod N)}^{n} u_{n}, \\
x_{n+1}=\left(1-\alpha_{n}\right) y_{n}+\alpha_{n} S_{n(\bmod N)}^{n} y_{n},
\end{array}\right.
$$

where $\left\{\alpha_{n}\right\}$ is a sequence in $(0,1)$ with $\liminf _{n \rightarrow \infty} \alpha_{n}>0,\left\{\beta_{n}\right\}$ is a sequence in $(0,1-k)$ with $\liminf _{n \rightarrow \infty} \beta_{n}>0, k=\max \left\{\tau_{1}, \tau_{2}, \ldots, \tau_{N}\right\} \in(0,1)$, and the sequence $\left\{r_{n}\right\} \subset(0, \infty)$ satisfies that $\liminf _{n \rightarrow \infty} r_{n}>0$ and $\lim _{n \rightarrow \infty}\left|r_{n+1}-r_{n}\right|=0$. If $\Gamma:=\bigcap_{i=1}^{N} F\left(S_{i}\right) \bigcap_{i=1}^{N} F\left(T_{i}\right) \cap$ $\operatorname{MEP}(F, \varphi) \neq \emptyset$, then the sequence $\left\{x_{n}\right\}$ converges weakly to a point $x^{*} \in \Gamma$.

Proof The proof is divided into four steps.

Step 1. Firstly, we prove that $\lim _{n \rightarrow \infty}\left\|x_{n}-p\right\|$ exists for any $p \in \Gamma$.

Taking $p \in \Gamma$ and putting $\rho=\max \left\{l_{1}, l_{2}, \ldots, l_{N}\right\} \in(0,1)$, it follows from Lemma 2.1 that $u_{n}=T_{r_{n}} x_{n}, p=T_{r_{n}} p$, we have

$$
\begin{aligned}
\left\|u_{n}-p\right\| \leq & \left\|T_{r_{n}} x_{n}-T_{r_{n}} p\right\| \leq\left\|x_{n}-p\right\|, \\
\left\|x_{n+1}-p\right\|^{2} & =\left\|y_{n}-p+\alpha_{n}\left(S_{n(\bmod N)}^{n} y_{n}-y_{n}\right)\right\|^{2} \\
& =\left\|y_{n}-p\right\|^{2}+2 \alpha_{n}\left(y_{n}-p, S_{n(\bmod N)}^{n} y_{n}-y_{n}\right\rangle+\alpha_{n}^{2}\left\|y_{n}-S_{n(\bmod N)}^{n} y_{n}\right\|^{2} .
\end{aligned}
$$

Since

$$
\begin{aligned}
\left\|S_{n(\bmod N)}^{n} y_{n}-p\right\|^{2} & =\left\|S_{n(\bmod N)}^{n} y_{n}-S_{n(\bmod N)}^{n} p\right\|^{2} \\
& \leq \rho_{n}\left\|y_{n}-p\right\|^{2}+\rho\left\|\left(I-S_{n(\bmod N)}^{n}\right) y_{n}\right\|^{2}
\end{aligned}
$$

and

$$
\begin{aligned}
\left\|S_{n(\bmod N)}^{n} y_{n}-p\right\|^{2}= & \left\|S_{n(\bmod N)}^{n} y_{n}-y_{n}+y_{n}-p\right\|^{2} \\
= & \left\|S_{n(\bmod N)}^{n} y_{n}-y_{n}\right\|^{2}+\left\|y_{n}-p\right\|^{2} \\
& +2\left\langle S_{n(\bmod N)}^{n} y_{n}-y_{n}, y_{n}-p\right\rangle,
\end{aligned}
$$

from (3.4) and (3.5) we have

$$
2\left\langle S_{n(\bmod N)}^{n} y_{n}-y_{n}, y_{n}-p\right\rangle \leq\left(\rho_{n}-1\right)\left\|y_{n}-p\right\|^{2}+(\rho-1)\left\|S_{n(\bmod N)}^{n} y_{n}-y_{n}\right\|^{2} .
$$


Substituting (3.6) into (3.3) and simplifying, we have

$$
\begin{aligned}
\left\|x_{n+1}-p\right\|^{2} \leq & \left\|y_{n}-p\right\|^{2}+\alpha_{n}\left(\rho_{n}-1\right)\left\|y_{n}-p\right\|^{2} \\
& +\alpha_{n}(\rho-1)\left\|S_{n(\bmod N)}^{n} y_{n}-y_{n}\right\|^{2}+\alpha_{n}^{2}\left\|y_{n}-S_{n(\bmod N)}^{n} y_{n}\right\|^{2} \\
= & {\left[1+\alpha_{n}\left(\rho_{n}-1\right)\right]\left\|y_{n}-p\right\|^{2}-\alpha_{n}\left(1-\rho-\alpha_{n}\right)\left\|S_{n(\bmod N)}^{n} y_{n}-y_{n}\right\|^{2} . }
\end{aligned}
$$

On the other hand,

$$
\begin{aligned}
\left\|y_{n}-p\right\|^{2} \leq & \left\|u_{n}-p\right\|^{2}+2 \beta_{n}\left\langle u_{n}-p,\left(T_{n(\bmod N)}^{n}-I\right) u_{n}\right\rangle \\
& \left.+\beta_{n}^{2} \| T_{n(\bmod N)}^{n}-I\right) u_{n} \|^{2} \\
= & \left\|u_{n}-p\right\|^{2}+2 \beta_{n}\left\langle u_{n}-p,\left(T_{n(\bmod N)}^{n}-I\right) u_{n}\right\rangle \\
& \left.+\beta_{n}^{2} \| T_{n(\bmod N)}^{n}-I\right) u_{n} \|^{2} .
\end{aligned}
$$

Since $T_{i}$ is a $\tau_{i}$-asymptotically strictly pseudononspreading mapping, we have

$$
\left\|T_{n(\bmod N)}^{n} u_{n}-p\right\|^{2} \leq k_{n}\left\|u_{n}-p\right\|^{2}+k\left\|u_{n}-T_{n(\bmod N)}^{n} u_{n}\right\|^{2}
$$

Again since

$$
\begin{aligned}
\left\|T_{n(\bmod N)}^{n} u_{n}-p\right\|^{2}= & \left\|T_{n(\bmod N)}^{n} u_{n}-u_{n}\right\|^{2}+\left\|u_{n}-p\right\|^{2} \\
& +2\left\langle T_{n(\bmod N)}^{n} u_{n}-u_{n}, u_{n}-p\right\rangle
\end{aligned}
$$

so we have

$$
\begin{aligned}
& 2\left\langle T_{n(\bmod N)}^{n} u_{n}-u_{n} \cdot u_{n}-p\right\rangle \\
& \quad \leq\left(k_{n}-1\right)\left\|u_{n}-p\right\|^{2}+(k-1)\left\|u_{n}-T_{n(\bmod N)}^{n} u_{n}\right\|^{2} .
\end{aligned}
$$

From (3.8) and (3.11), we have

$$
\begin{aligned}
\left\|y_{n}-p\right\|^{2} \leq & \left\|u_{n}-p\right\|^{2}+\beta_{n}\left(k_{n}-1\right)\left\|u_{n}-p\right\|^{2}+\beta_{n}(k-1)\left\|u_{n}-T_{n(\bmod N)}^{n} u_{n}\right\|^{2} \\
& \left.+\beta_{n}^{2} \| T_{n(\bmod N)}^{n}-I\right) u_{n} \|^{2} \\
\leq & {\left[1+\beta_{n}\left(k_{n}-1\right)\right]\left\|u_{n}-p\right\|^{2}-\beta_{n}\left(1-k-\beta_{n}\right)\left\|u_{n}-T_{n(\bmod N)}^{n} u_{n}\right\|^{2} . }
\end{aligned}
$$

By using (3.7) and (3.12), we have

$$
\begin{aligned}
\left\|x_{n+1}-p\right\|^{2} \leq & {\left[1+\alpha_{n}\left(\rho_{n}-1\right)\right]\left\{\left[1+\beta_{n}\left(k_{n}-1\right)\right]\left\|u_{n}-p\right\|^{2}\right.} \\
& \left.-\beta_{n}\left(1-k-\beta_{n}\right)\left\|u_{n}-T_{n(\bmod N)}^{n} u_{n}\right\|^{2}\right\} \\
& -\alpha_{n}\left(1-\rho-\alpha_{n}\right)\left\|S_{n(\bmod N)}^{n} y_{n}-y_{n}\right\|^{2} \\
= & {\left[1+\alpha_{n}\left(\rho_{n}-1\right)\right]\left[1+\beta_{n}\left(k_{n}-1\right)\right]\left\|u_{n}-p\right\|^{2} } \\
& -\beta_{n}\left[1+\alpha_{n}\left(\rho_{n}-1\right)\right]\left(1-k-\beta_{n}\right)\left\|u_{n}-T_{n(\bmod N)}^{n} u_{n}\right\|^{2}
\end{aligned}
$$




$$
\begin{aligned}
& -\alpha_{n}\left(1-\rho-\alpha_{n}\right)\left\|S_{n(\bmod N)}^{n} y_{n}-y_{n}\right\|^{2} \\
\leq & \left\{1+\beta_{n}\left(k_{n}-1\right)+\alpha_{n}\left(\rho_{n}-1\right)\left[1+\beta_{n}\left(k_{n}-1\right)\right]\right\}\left\|x_{n}-p\right\|^{2} \\
& -\beta_{n}\left[1+\alpha_{n}\left(\rho_{n}-1\right)\right]\left(1-k-\beta_{n}\right)\left\|u_{n}-T_{n(\bmod N)}^{n} u_{n}\right\|^{2} \\
& -\alpha_{n}\left(1-\rho-\alpha_{n}\right)\left\|S_{n(\bmod N)}^{n} y_{n}-y_{n}\right\|^{2} .
\end{aligned}
$$

Let $M_{n}:=\beta_{n}\left(k_{n}-1\right)+\alpha_{n}\left(\rho_{n}-1\right)\left[1+\beta_{n}\left(k_{n}-1\right)\right]$. Since $\sum\left(\rho_{n}-1\right)<\infty$ and $\sum\left(k_{n}-1\right)<\infty$, so $\sum M_{n}<\infty$, we have

$$
\begin{aligned}
\left\|x_{n+1}-p\right\|^{2} \leq & \left(1+M_{n}\right)\left\|x_{n}-p\right\|^{2}-\alpha_{n}\left(1-\rho-\alpha_{n}\right)\left\|S_{n(\bmod N)}^{n} y_{n}-y_{n}\right\|^{2} \\
& -\beta_{n}\left[1+\alpha_{n}\left(\rho_{n}-1\right)\right]\left(1-k-\beta_{n}\right)\left\|u_{n}-T_{n(\bmod N)}^{n} u_{n}\right\|^{2} \\
\leq & \left(1+M_{n}\right)\left\|x_{n}-p\right\|^{2} .
\end{aligned}
$$

Using Lemma 2.5, we show that $\lim _{n \rightarrow \infty}\left\|x_{n}-p\right\|$ exists. Further, it follows from (3.2) and (3.12) that $\left\{y_{n}\right\}$ and $\left\{u_{n}\right\}$ are bounded.

On the other hand, from (3.14) we have

$$
\begin{aligned}
& \beta_{n}\left[1+\alpha_{n}\left(\rho_{n}-1\right)\right]\left(1-k-\beta_{n}\right)\left\|u_{n}-T_{n(\bmod N)}^{n} u_{n}\right\|^{2}+\alpha_{n}\left(1-\rho-\alpha_{n}\right)\left\|S_{n(\bmod N)}^{n} y_{n}-y_{n}\right\|^{2} \\
& \quad \leq\left(1+M_{n}\right)\left\|x_{n}-p\right\|^{2}-\left\|x_{n+1}-p\right\|^{2} .
\end{aligned}
$$

Since $\lim _{n \rightarrow \infty}\left\|x_{n}-p\right\|$ exists and by the fact that $M_{n} \rightarrow 0$, taking limit on both sides of inequality (3.15), we get

$$
\begin{aligned}
& \lim _{n \rightarrow \infty}\left\|\left(T_{n(\bmod N)}^{n}-I\right) u_{n}\right\|=0, \\
& \lim _{n \rightarrow \infty}\left\|y_{n}-S_{n(\bmod N)}^{n} y_{n}\right\|=0 .
\end{aligned}
$$

Step 2. Now, we prove that $\lim _{n \rightarrow \infty}\left\|x_{n+1}-x_{n}\right\|=0, \lim _{n \rightarrow \infty}\left\|y_{n+1}-y_{n}\right\|=0$ and $\lim _{n \rightarrow \infty}\left\|x_{n}-u_{n}\right\|=0$.

It follows from Lemma 2.1 that $u_{n}=T_{r_{n}} x_{n}, p=T_{r_{n}} p$, so

$$
\begin{aligned}
\left\|u_{n}-p\right\|^{2} & =\left\|T_{r_{n}} x_{n}-T_{r_{n}} p\right\|^{2} \\
& \leq\left\langle x_{n}-p, u_{n}-p\right\rangle \\
& =\frac{1}{2}\left(\left\|x_{n}-p\right\|^{2}+\left\|u_{n}-p\right\|^{2}-\left\|x_{n}-u_{n}\right\|^{2}\right) .
\end{aligned}
$$

This shows that

$$
\left\|u_{n}-p\right\|^{2} \leq\left\|x_{n}-p\right\|^{2}-\left\|x_{n}-u_{n}\right\|^{2} .
$$

By (3.13) and (3.19), we obtain

$$
\left\|x_{n+1}-p\right\|^{2} \leq\left\|x_{n}-p\right\|^{2}-\left\|x_{n}-u_{n}\right\|^{2}+M_{n}\left\|x_{n}-p\right\|^{2} .
$$


So,

$$
\left\|x_{n}-u_{n}\right\|^{2} \leq\left\|x_{n}-q\right\|^{2}-\left\|x_{n+1}-q\right\|^{2}+M_{n}\left\|x_{n}-p\right\|^{2} .
$$

Thus, we obtain

$$
\lim _{n \rightarrow \infty}\left\|x_{n}-u_{n}\right\|=0
$$

In fact, it follows from (3.1) that

$$
\begin{aligned}
\left\|x_{n+1}-x_{n}\right\|= & \left\|\left(1-\alpha_{n}\right) y_{n}+\alpha_{n} S_{n(\bmod N)}^{n} y_{n}-x_{n}\right\| \\
= & \left\|\left(1-\alpha_{n}\right)\left(u_{n}+\beta_{n}\left(T_{n(\bmod N)}^{n}-I\right) u_{n}\right)+\alpha_{n} S_{n(\bmod N)}^{n} y_{n}-x_{n}\right\| \\
= & \left\|\left(1-\alpha_{n}\right) \beta_{n}\left(T_{n(\bmod N)}^{n}-I\right) u_{n}+\alpha_{n}\left(S_{n(\bmod N)}^{n} y_{n}-u_{n}\right)+\left(u_{n}-x_{n}\right)\right\| \\
= & \|\left(1-\alpha_{n}\right) \beta_{n}\left(T_{n(\bmod N)}^{n}-I\right) u_{n}+\alpha_{n}\left(S_{n(\bmod N)}^{n} y_{n}-y_{n}\right) \\
& +\alpha_{n}\left(y_{n}-u_{n}\right)+\left(u_{n}-x_{n}\right) \| \\
= & \|\left(1-\alpha_{n}\right) \beta_{n}\left(T_{n(\bmod N)}^{n}-I\right) u_{n}+\alpha_{n}\left(S_{n(\bmod N)}^{n} y_{n}-y_{n}\right) \\
& +\alpha_{n} \beta_{n}\left(T_{n(\bmod N)}^{n}-I\right) u_{n}+\left(u_{n}-x_{n}\right) \| \\
= & \left\|\beta_{n}\left(T_{n(\bmod N)}^{n}-I\right) u_{n}+\alpha_{n}\left(S_{n(\bmod N)}^{n} y_{n}-y_{n}\right)\right\|+\left\|u_{n}-x_{n}\right\| \\
\leq & \left\|\beta_{n}\left(T_{n(\bmod N)}^{n}-I\right) u_{n}\right\|+\alpha_{n}\left\|S_{n(\bmod N)}^{n} y_{n}-y_{n}\right\|+\left\|u_{n}-x_{n}\right\| .
\end{aligned}
$$

From (3.16), (3.17) and (3.22) we have

$$
\lim _{n \rightarrow \infty}\left\|x_{n+1}-x_{n}\right\|=0 .
$$

Similarly, it follows from (3.1) that

$$
\begin{aligned}
\left\|y_{n+1}-y_{n}\right\|= & \left\|u_{n+1}+\beta_{n+1}\left(T_{n+1(\bmod N)}^{n+1}-I\right) u_{n+1}-u_{n}+\beta_{n}\left(T_{n(\bmod N)}^{n}-I\right) u_{n}\right\| \\
\leq & \left\|u_{n+1}-u_{n}\right\|+\beta_{n+1}\left\|\left(T_{n+1(\bmod N)}^{n+1}-I\right) u_{n+1}\right\| \\
& +\beta_{n}\left\|\left(T_{n(\bmod N)}^{n}-I\right) u_{n}\right\|,
\end{aligned}
$$

where

$$
\begin{aligned}
\left\|u_{n+1}-u_{n}\right\| & =\left\|T_{r_{n+1}} x_{n+1}-T_{r_{n}} x_{n}\right\| \\
& \leq\left\|T_{r_{n+1}} x_{n+1}-T_{r_{n+1}} x_{n}\right\|+\left\|T_{r_{n+1}} x_{n}-T_{r_{n}} x_{n}\right\| \\
& \leq\left\|x_{n+1}-x_{n}\right\|+\left\|T_{r_{n+1}} x_{n}-T_{r_{n}} x_{n}\right\| .
\end{aligned}
$$

On the other hand, it follows from Lemma 2.1 that $u_{n}=T_{r_{n}} x_{n}$ and $u_{n+1}=T_{r_{n+1}} x_{n+1}$. We have

$$
F\left(u_{n+1}, y\right)+\varphi(y)-\varphi\left(u_{n+1}\right)+\frac{1}{r_{n+1}}\left\langle y-u_{n+1}, u_{n+1}-x_{n+1}\right\rangle \geq 0, \quad \forall y \in C,
$$


and

$$
F\left(u_{n}, y\right)+\varphi(y)-\varphi\left(u_{n}\right)+\frac{1}{r_{n}}\left\langle y-u_{n}, u_{n}-x_{n}\right\rangle \geq 0, \quad \forall y \in C .
$$

Particularly, we have

$$
F\left(u_{n+1}, u_{n}\right)+\varphi\left(u_{n}\right)-\varphi\left(u_{n+1}\right)+\frac{1}{r_{n+1}}\left\langle u_{n}-u_{n+1}, u_{n+1}-x_{n+1}\right\rangle \geq 0, \quad \forall y \in C,
$$

and

$$
F\left(u_{n}, u_{n+1}\right)+\varphi\left(u_{n+1}\right)-\varphi\left(u_{n}\right)+\frac{1}{r_{n}}\left\langle u_{n+1}-u_{n}, u_{n}-x_{n}\right\rangle \geq 0, \quad \forall y \in C .
$$

Summing up (3.27) and (3.28) and using (A2), we obtain

$$
\frac{1}{r_{n+1}}\left\langle u_{n}-u_{n+1}, u_{n+1}-x_{n+1}\right\rangle+\frac{1}{r_{n}}\left\langle u_{n+1}-u_{n}, u_{n}-x_{n}\right\rangle \geq 0 .
$$

Thus,

$$
\left\langle u_{n+1}-u_{n}, \frac{u_{n}-x_{n}}{r_{n}}-\frac{u_{n+1}-x_{n+1}}{r_{n+1}}\right\rangle \geq 0
$$

which implies that

$$
\begin{aligned}
0 & \leq\left\langle u_{n+1}-u_{n}, u_{n}-x_{n}-\frac{r_{n}}{r_{n+1}}\left(u_{n+1}-x_{n+1}\right)\right\rangle \\
& =\left\langle u_{n+1}-u_{n}, u_{n}-u_{n+1}+u_{n+1}-x_{n}-\frac{r_{n}}{r_{n+1}}\left(u_{n+1}-x_{n+1}\right)\right\rangle .
\end{aligned}
$$

Therefore,

$$
\begin{aligned}
\left\|u_{n+1}-u_{n}\right\|^{2} & \leq\left\langle u_{n+1}-u_{n}, x_{n+1}-x_{n}+\left(1-\frac{r_{n}}{r_{n+1}}\right)\left(u_{n+1}-x_{n+1}\right)\right\rangle \\
& \leq\left\|u_{n+1}-u_{n}\right\| \cdot\left[\left\|x_{n+1}-x_{n}\right\|+\left|1-\frac{r_{n}}{r_{n+1}}\right| \cdot\left\|u_{n+1}-x_{n+1}\right\|\right] .
\end{aligned}
$$

Thus, we have

$$
\left\|u_{n+1}-u_{n}\right\| \leq\left\|x_{n+1}-x_{n}\right\|+\left|1-\frac{r_{n}}{r_{n+1}}\right| \cdot\left\|u_{n+1}-x_{n+1}\right\| .
$$

It follows from (3.16), (3.17), (3.24), (3.25) and (3.29) that

$$
\lim _{n \rightarrow \infty}\left\|u_{n+1}-u_{n}\right\|=0
$$

and

$$
\lim _{n \rightarrow \infty}\left\|y_{n+1}-y_{n}\right\|=0
$$


Put $L=\left\{L_{1}, L_{2}, \ldots, L_{N}, \widetilde{L}_{1}, \widetilde{L}_{2}, \ldots, \widetilde{L}_{N}\right\}$. Since

$$
\begin{aligned}
\left\|u_{n}-T_{n(\bmod N)} u_{n}\right\| \leq & \left\|u_{n}-T_{n(\bmod N)}^{n} u_{n}\right\|+\left\|T_{n(\bmod N)}^{n} u_{n}-T_{n(\bmod N)} u_{n}\right\| \\
\leq & \left\|u_{n}-T_{n(\bmod N)}^{n} u_{n}\right\|+L\left\|T_{n(\bmod N)}^{n-1} u_{n}-u_{n}\right\| \\
\leq & \left\|u_{n}-T_{n(\bmod N)}^{n} u_{n}\right\|+L\left[\left\|T_{n-1(\bmod N)}^{n-1} u_{n}-T_{n-1(\bmod N)}^{n-1} u_{n-1}\right\|\right. \\
& \left.\quad+\left\|T_{n-1(\bmod N)}^{n-1} u_{n-1}-u_{n-1}\right\|+\left\|u_{n-1}-u_{n}\right\|\right] \\
\leq & \left\|u_{n}-T_{n(\bmod N)}^{n} u_{n}\right\|+L^{2}\left\|u_{n}-u_{n-1}\right\| \\
& +L\left\|T_{n-1(\bmod N)}^{n-1} u_{n-1}-u_{n-1}\right\|+L\left\|u_{n-1}-u_{n}\right\|,
\end{aligned}
$$

from (3.16), (3.22), (3.30) and (3.32) we get

$$
\lim _{n \rightarrow \infty}\left\|T_{n(\bmod N)} u_{n}-u_{n}\right\|=0
$$

Similarly, we have

$$
\begin{aligned}
\left\|y_{n}-S_{n(\bmod N)} y_{n}\right\| \leq & \left\|y_{n}-S_{n(\bmod N)}^{n} y_{n}\right\|+\left\|S_{n(\bmod N)}^{n} y_{n} y_{n}-S_{n(\bmod N)} y_{n}\right\| \\
\leq & \left\|y_{n}-S_{n(\bmod N)}^{n} y_{n}\right\|+L\left[\left\|S_{n-1(\bmod N)}^{n-1} y_{n}-S_{n-1(\bmod N)}^{n-1} y_{n-1}\right\|\right. \\
& \left.\quad+\left\|S_{n-1(\bmod N)}^{n-1} y_{n-1}-y_{n-1}\right\|+\left\|y_{n-1}-y_{n}\right\|\right] \\
\leq & \left\|y_{n}-T_{n(\bmod N)}^{n} y_{n}\right\|+L^{2}\left\|y_{n}-y_{n-1}\right\| \\
& +L\left\|T_{n-1(\bmod N)}^{n-1} y_{n-1}-y_{n-1}\right\|+L\left\|y_{n-1}-y_{n}\right\| .
\end{aligned}
$$

This implies that

$$
\lim _{n \rightarrow \infty}\left\|y_{n}-S_{n(\bmod N)} y_{n}\right\|=0 .
$$

Since $\left\|x_{n+1}-y_{n}\right\|=\alpha_{n}\left\|y_{n}-S_{n(\bmod N)}^{n} y_{n}\right\|$, so

$$
\lim _{n \rightarrow \infty}\left\|x_{n+1}-y_{n}\right\|=0 .
$$

By (3.24) and (3.35), we have

$$
\lim _{n \rightarrow \infty}\left\|x_{n}-y_{n}\right\|=0
$$

It follows from (3.22) and (3.36) that

$$
\lim _{n \rightarrow \infty}\left\|y_{n}-u_{n}\right\|=0
$$

Since $\lim _{n \rightarrow \infty}\left\|x_{n}-p\right\|$ exists for any $p \in \Gamma$ and $\left\|x_{n}-p\right\|-\left\|x_{n}-y_{n}\right\| \leq\left\|y_{n}-p\right\| \leq\left\|x_{n}-p\right\|+$ $\left\|x_{n}-y_{n}\right\|$, it follows from (3.36) that $\lim _{n \rightarrow \infty}\left\|y_{n}-p\right\|=\lim _{n \rightarrow \infty}\left\|x_{n}-p\right\|$ holds. Similarly, $\lim _{n \rightarrow \infty}\left\|u_{n}-p\right\|=\lim _{n \rightarrow \infty}\left\|x_{n}-p\right\|$ holds for any $p \in \Gamma$.

Step 3. We show that $x^{*} \in \Gamma:=\bigcap_{i=1}^{N} F\left(S_{i}\right) \bigcap_{i=1}^{N} F\left(T_{i}\right) \cap \operatorname{MEP}(F, \varphi)$.

Firstly, we show that $x^{*} \in \bigcap_{i=1}^{N} F\left(S_{i}\right) \bigcap_{i=1}^{N} F\left(T_{i}\right)$. 
In fact, since $\left\{y_{n}\right\}$ is bounded, there exists a subsequence $\left\{y_{n_{i}}\right\} \subset\left\{y_{n}\right\}$ such that $\left\{y_{n_{i}}\right\} \rightarrow$ $x^{*} \in C$. Hence, for any positive integer $j=1,2, \ldots, N$, there exists a subsequence $\left\{n_{i}(j)\right\} \subset$ $\left\{n_{i}\right\}$ with $n_{i}(j)(\bmod N)=j$ such that $\left\{y_{n_{i}(j)}\right\} \rightarrow x^{*}$. Again, by (3.34) we know that $\| y_{i N+j}-$ $S_{j} u_{i N+j} \| \rightarrow 0$ as $i \rightarrow \infty$, therefore we have that $\lim _{n_{i(j)} \rightarrow \infty}\left\|y_{n_{i}(j)}-S_{j} y_{n_{i}(j)}\right\|=0$.

Since $S_{j}$ is demiclosed at zero, it follows from Lemma 2.4 that $x^{*} \in F\left(S_{j}\right)$. By the arbitrariness of $j=1,2, \ldots, N$, we have

$$
x^{*} \in \bigcap_{i=1}^{N} F\left(S_{i}\right) \text {. }
$$

On the other hand, since $\lim _{n \rightarrow \infty}\left\|y_{n}-u_{n}\right\|=0$, we know that $u_{n_{i}} \rightarrow x^{*}$, too. Similarly, it follows from (3.33) and Lemma 2.4 that $x^{*} \in F\left(T_{j}\right)$. By the arbitrariness of $j=1,2, \ldots, N$, we have

$$
x^{*} \in \bigcap_{i=1}^{N} F\left(T_{i}\right) .
$$

Now, we show that $x^{*} \in \operatorname{MEP}(F, \varphi)$.

By Lemma 2.1, since $u_{n}=T_{r_{n}} x_{n}$, we have

$$
F\left(u_{n}, y\right)+\varphi(y)-\varphi\left(u_{n}\right)+\frac{1}{r_{n}}\left\langle y-u_{n}, u_{n}-x_{n}\right\rangle \geq 0, \quad \forall y \in K .
$$

From (A2), we obtain

$$
\varphi(y)-\varphi\left(u_{n}\right)+\frac{1}{r_{n}}\left\langle y-u_{n}, u_{n}-x_{n}\right\rangle \geq-F\left(u_{n}, y\right) \geq F\left(y, u_{n}\right),
$$

and hence

$$
\varphi(y)-\varphi\left(u_{n_{i}}\right)+\frac{1}{r_{n_{i}}}\left\langle y-u_{n_{i}}, u_{n_{i}}-x_{n_{i}}\right\rangle \geq F\left(y, u_{n_{i}}\right) .
$$

By $\liminf n_{n \rightarrow \infty} r_{n}>0$, we have $\lim _{i \rightarrow \infty} \frac{\left\|u_{n_{i}}-x_{n_{i}}\right\|}{r_{n_{i}}}=0$. Since $u_{n_{i}} \rightarrow x^{*}$, it follows from (A4) and the weak lower semicontinuity of $\varphi$ that

$$
F\left(y, x^{*}\right)-\varphi(y)+\varphi\left(x^{*}\right) \leq 0 .
$$

Put $z_{t}=t y+(1-t) x^{*}$ for all $t \in(0,1]$ and $y \in C$. Consequently, we get $z_{t} \in C$. Hence

$$
F\left(z_{t}, p\right)-\varphi\left(z_{t}\right)+\varphi\left(x^{*}\right) \leq 0 .
$$

From (A1) and (A4), and the convexity of $\varphi$, we have

$$
\begin{aligned}
0 & =F\left(z_{t}, z_{t}\right)-\varphi\left(z_{t}\right)+\varphi\left(z_{t}\right) \\
& \leq t F\left(z_{t}, y\right)+(1-t) \varphi\left(z_{t}, x^{*}\right)+t \varphi(y)+(1-t) \varphi\left(x^{*}\right)-\varphi\left(z_{t}\right) \\
& \leq t\left[F\left(z_{t}, y\right)+\varphi(y)-\varphi\left(z_{t}\right)\right] .
\end{aligned}
$$


Therefore

$$
F\left(z_{t}, y\right)+\varphi(y)-\varphi\left(z_{t}\right) \geq 0
$$

Letting $t \rightarrow 0$, and from the weak lower semicontinuity of $\varphi$, we have

$$
F\left(x^{*}, y\right)+\varphi(y)-\varphi\left(x^{*}\right) \geq 0
$$

This implies that $x^{*} \in \operatorname{MEP}(F, \varphi)$. Hence $x^{*} \in \Gamma$.

Step 4. Finally, we prove that $x_{n} \rightarrow x^{*}$ and $u_{n} \rightarrow x^{*}, x^{*} \in \Gamma$.

Due to $u_{n_{i}} \rightarrow x^{*}$, we know that $x_{n_{i}} \rightarrow x^{*}$ from (3.37). Suppose that there exists another subsequence $\left\{x_{n_{j}}\right\}$ of $\left\{x_{n}\right\}$ such that $\left\{x_{n_{j}}\right\} \rightarrow y^{*} \in \Gamma$ with $y^{*} \neq x^{*}$. Using the same proof method as in Step 3, we know that $y^{*} \in \Gamma$. Consequently, $\lim _{n \rightarrow \infty}\left\|x_{n}-y^{*}\right\|$ exists. By using Opial's property of a Hilbert space, we have

$$
\begin{aligned}
\liminf _{n_{i} \rightarrow \infty}\left\|x_{n_{i}}-x^{*}\right\| & <\liminf _{n_{i} \rightarrow \infty}\left\|x_{n_{i}}-y^{*}\right\|=\liminf _{n \rightarrow \infty}\left\|x_{n}-y^{*}\right\| \\
& =\liminf _{n_{j} \rightarrow \infty}\left\|x_{n_{j}}-y^{*}\right\|<\liminf _{n_{j} \rightarrow \infty}\left\|x_{n_{j}}-x^{*}\right\| \\
& =\liminf _{n \rightarrow \infty}\left\|x_{n}-x^{*}\right\|=\liminf _{n_{i} \rightarrow \infty}\left\|x_{n_{i}}-x^{*}\right\| .
\end{aligned}
$$

This is a contradiction. Therefore $x_{n} \rightarrow x^{*}$. By (3.1) and (3.22), we have $u_{n} \rightarrow x^{*}$. Therefore, the conclusion follows.

This completes the proof of Theorem 3.1.

Taking $\varphi=0, N=1$ in Theorem 3.1, we have the following result.

Corollary 3.2 Let $C$ be a nonempty and closed convex subset of a real Hilbert space H, let $F$ be a bifunction from $C \times C$ to $R$ satisfying (A1)-(A4), and let $T: C \rightarrow C$ be a uniformly $L$ Lipschitzian and $k$-asymptotically strictly pseudononspreading mapping with the sequence $\left\{k_{n}\right\} \subset[1,+\infty)$ such that $\sum_{n=1}^{\infty}\left(k_{n}-1\right)<\infty$, let $S: C \rightarrow C$ be a uniformly $\widetilde{L}$-Lipschitzian and $\rho$-asymptotically strictly pseudononspreading mapping with the sequence $\left\{\rho_{n}\right\} \subset[1,+\infty)$ such that $\sum_{n=1}^{\infty}\left(\rho_{n}-1\right)<\infty$. Let $\left\{x_{n}\right\}$ be a sequence generated by

$$
\left\{\begin{array}{l}
\forall x_{1} \in C, \\
F\left(u_{n}, y\right)+\frac{1}{r_{n}}\left\langle y-u_{n}, u_{n}-x_{n}\right\rangle \geq 0, \quad \forall y \in C, \\
y_{n}=\left(1-\beta_{n}\right) u_{n}+\beta_{n} T^{n} u_{n}, \\
x_{n+1}=\left(1-\alpha_{n}\right) y_{n}+\alpha_{n} S^{n} y_{n},
\end{array}\right.
$$

where $k \in(0,1), \rho \in(0,1),\left\{\alpha_{n}\right\}$ is a sequence in $(0,1)$ with $\liminf _{n \rightarrow \infty} \alpha_{n}>0,\left\{\beta_{n}\right\}$ is a sequence in $(0,1-k)$ with $\liminf _{n \rightarrow \infty} \beta_{n}>0$ and the sequence $\left\{r_{n}\right\} \subset(0, \infty)$ with $\liminf _{n \rightarrow \infty} r_{n}>0$ and $\lim _{n \rightarrow \infty}\left|r_{n+1}-r_{n}\right|=0$. If $\Gamma:=F(S) \cap F(T) \cap E P(F) \neq \emptyset$, then the sequence $\left\{x_{n}\right\}$ converges weakly to a point $x^{*} \in \Gamma$.

Corollary 3.3 Let $C$ be a nonempty and closed convex subset of a real Hilbert space $H$, let $F$ be a bifunction from $C \times C$ to $R$ satisfying (A1)-(A4), and let $\varphi: C \rightarrow R \cup\{+\infty\}$ be a proper lower semi-continuous and convex function such that $C \cap \operatorname{dom} \varphi \neq \emptyset$. Let $T_{i}$ : 
$C \rightarrow C$ be a uniformly $L_{i}$-Lipschitzian and $\tau_{i}$-asymptotically strictly pseudononspreading mapping with the sequence $\left\{k_{n}\right\} \subset[1,+\infty)$ such that $\sum_{n=1}^{\infty}\left(k_{n}-1\right)<\infty$, let $S_{i}: C \rightarrow C$ be a uniformly $\widetilde{L}_{i}$-Lipschitzian and $l_{i}$-asymptotically strictly pseudononspreading mapping with the sequence $\left\{\rho_{n}\right\} \subset[1,+\infty)$ such that $\sum_{n=1}^{\infty}\left(\rho_{n}-1\right)<\infty, i=1,2, \ldots, N$. Let $\left\{x_{n}\right\}$ be a sequence generated by

$$
\left\{\begin{array}{l}
\forall x_{1} \in C, \\
F\left(u_{n}, y\right)+\varphi(y)-\varphi\left(u_{n}\right)+\frac{1}{r_{n}}\left\langle y-u_{n}, u_{n}-x_{n}\right\rangle \geq 0, \quad \forall y \in C, \\
y_{n}=\left(1-\beta_{n}\right) u_{n}+\beta_{n} T_{n(\bmod N)}^{n} u_{n}, \\
x_{n+1}=\left(1-\alpha_{n}\right) y_{n}+\alpha_{n} S_{n(\bmod N)} y_{n},
\end{array}\right.
$$

where $k=\max \left\{\tau_{1}, \tau_{2}, \ldots, \tau_{N}\right\} \in(0,1), \rho=\max \left\{l_{1}, l_{2}, \ldots, l_{N}\right\} \in(0,1),\left\{\alpha_{n}\right\}$ is a sequence in $(0,1)$ with $\liminf _{n \rightarrow \infty} \alpha_{n}>0,\left\{\beta_{n}\right\}$ is a sequence in $(0,1-k)$ with $\liminf _{n \rightarrow \infty} \beta_{n}>0$ and the sequence $\left\{r_{n}\right\} \subset(0, \infty)$ with $\liminf _{n \rightarrow \infty} r_{n}>0$ and $\lim _{n \rightarrow \infty}\left|r_{n+1}-r_{n}\right|=0$. If $\Gamma:=$ $\bigcap_{i=1}^{\infty} F\left(S_{i}\right) \bigcap_{i=1}^{\infty} F\left(T_{i}\right) \cap \operatorname{MEP}(F, \varphi) \neq \emptyset$, and there exists a positive integer $j$ such that $S_{j}$ is semi-compact, then the sequence $\left\{x_{n}\right\}$ converges strongly to a point $x^{*} \in \Gamma$.

Proof Without loss of generality, we can assume that $S_{1}$ is semi-compact. It follows from (3.34) that

$$
\left\|y_{n_{i}(1)}-S_{1} y_{n_{i}(1)}\right\| \rightarrow 0, \quad n_{i(1)} \rightarrow \infty
$$

Therefore, there exists a subsequence of $\left\{y_{n_{i}(1)}\right\}$ (for the sake of convenience we still denote it by $\left.\left\{y_{n_{i}(1)}\right\}\right)$ such that $y_{n_{i}(1)} \rightarrow y^{*} \in H_{1}$. Since $y_{n_{i}(1)} \rightarrow y^{*}, x^{*}=y^{*}$, and so $y_{n_{i}(1)} \rightarrow x^{*} \in \Gamma$. By virtue of the fact that $\lim _{n \rightarrow \infty}\left\|y_{n}-p\right\|$ exists, we know that

$$
\lim _{n \rightarrow \infty}\left\|y_{n}-x^{*}\right\|=\lim _{n \rightarrow \infty}\left\|u_{n}-x^{*}\right\|=\lim _{n \rightarrow \infty}\left\|x_{n}-x^{*}\right\|=0 .
$$

That is, $\left\{x_{n}\right\},\left\{u_{n}\right\}$ and $\left\{y_{n}\right\}$ converge strongly to the point $x^{*} \in \Gamma$. This completes the proof.

\section{Applications}

\subsection{Application to a convex minimization problem}

It is well known that mixed equilibrium problem (1.2) reduces to the convex minimization problem as $F=0$. Therefore, Theorem 3.1 can be used to solve convex minimization problem (1.3), and the following result can be directly deduced from Theorem 3.1.

Theorem 4.1 Let $C$ be a nonempty and closed convex subset of a real Hilbert space $H$, let $\varphi: C \rightarrow R \cup\{+\infty\}$ be a proper lower semi-continuous and convex function such that $C \cap \operatorname{dom} \varphi \neq \emptyset$. Let $T_{i}: C \rightarrow C$ be a uniformly $L_{i}$-Lipschitzian and $\tau_{i}$-asymptotically strictly pseudononspreading mapping with the sequence $\left\{k_{n}\right\} \subset[1,+\infty)$ such that $\sum_{n=1}^{\infty}\left(k_{n}-1\right)<\infty$, let $S_{i}: C \rightarrow C$ be a uniformly $\widetilde{L}_{i}$-Lipschitzian and $l_{i}$-asymptotically strictly pseudononspreading mapping with the sequence $\left\{\rho_{n}\right\} \subset[1,+\infty)$ such that $\sum_{n=1}^{\infty}\left(\rho_{n}-1\right)<\infty, i=$ 
$1,2, \ldots, N$. Let $\left\{x_{n}\right\}$ be a sequence generated by

$$
\left\{\begin{array}{l}
\forall x_{1} \in C, \\
\varphi(y)-\varphi\left(u_{n}\right)+\frac{1}{r_{n}}\left\langle y-u_{n}, u_{n}-x_{n}\right\rangle \geq 0, \quad \forall y \in C, \\
y_{n}=\left(1-\beta_{n}\right) u_{n}+\beta_{n} T_{n(\bmod N)}^{n} u_{n}, \\
x_{n+1}=\left(1-\alpha_{n}\right) y_{n}+\alpha_{n} S_{n(\bmod N)}^{n} y_{n},
\end{array}\right.
$$

where $k=\max \left\{\tau_{1}, \tau_{2}, \ldots, \tau_{N}\right\} \in(0,1), \rho=\max \left\{l_{1}, l_{2}, \ldots, l_{N}\right\} \in(0,1),\left\{\alpha_{n}\right\}$ is a sequence in $(0,1)$ with $\liminf _{n \rightarrow \infty} \alpha_{n}>0,\left\{\beta_{n}\right\}$ is a sequence in $(0,1-k)$ with $\liminf _{n \rightarrow \infty} \beta_{n}>0$, and the sequence $\left\{r_{n}\right\} \subset(0, \infty)$ with $\liminf _{n \rightarrow \infty} r_{n}>0$ and $\lim _{n \rightarrow \infty}\left|r_{n+1}-r_{n}\right|=0$. If $\bigcap_{i=1}^{N} F\left(S_{i}\right) \bigcap_{i=1}^{N} F\left(T_{i}\right) \cap C M P(\varphi) \neq \emptyset$, then the sequence $\left\{x_{n}\right\}$ converges weakly to a point $x^{*} \in \bigcap_{i=1}^{N} F\left(S_{i}\right) \bigcap_{i=1}^{N} F\left(T_{i}\right) \cap \operatorname{CMP}(\varphi)$.

\subsection{Application to a convex feasibility problem}

The so-called convex feasibility problem for a family of mappings $\left\{T_{i}\right\}_{i=1}^{\omega}$ (where $\omega$ may be a finite positive integer or $+\infty)$ is to find a point of the nonempty intersection $\bigcap_{i=1}^{\omega} C_{i}$, where $C_{i}$ is the fixed point set of mapping $T_{i}, i=1,2, \ldots, \omega$.

In Theorem 3.1 if $F=0, \varphi=0$, then the condition ' $u_{n} \in C$ such that $\forall y \in C,\left\langle y-u_{n}, u_{n}-\right.$ $\left.x_{n}\right\rangle \geq 0$ ' is equivalent to $u_{n}=P_{C}\left(x_{n}\right)$. Therefore, the following result can be directly obtained from Theorem 3.1.

Theorem 4.2 Let $C$ be a nonempty and closed convex subset of a real Hilbert space $H$, let $T_{i}: C \rightarrow C$ be a uniformly $L_{i}$-Lipschitzian and $\tau_{i}$-asymptotically strictly pseudononspreading mapping with the sequence $\left\{k_{n}\right\} \subset[1,+\infty)$ such that $\sum_{n=1}^{\infty}\left(k_{n}-1\right)<\infty$, let $S_{i}: C \rightarrow C$ be a uniformly $\widetilde{L}_{i}$-Lipschitzian and $l_{i}$-asymptotically strictly pseudononspreading mapping with the sequence $\left\{\rho_{n}\right\} \subset[1,+\infty)$ such that $\sum_{n=1}^{\infty}\left(\rho_{n}-1\right)<\infty, i=1,2, \ldots, N$. Let $\left\{x_{n}\right\}$ be a sequence generated by

$$
\left\{\begin{array}{l}
\forall x_{1} \in C \\
u_{n}=P_{C}\left(x_{n}\right) \\
y_{n}=\left(1-\beta_{n}\right) u_{n}+\beta_{n} T_{n(\bmod N)}^{n} u_{n}, \\
x_{n+1}=\left(1-\alpha_{n}\right) y_{n}+\alpha_{n} S_{n(\bmod N)}^{n} y_{n}
\end{array}\right.
$$

where $\left\{\alpha_{n}\right\}$ is a sequence in $(0,1)$ with $\liminf _{n \rightarrow \infty} \alpha_{n}>0$ and $\left\{\beta_{n}\right\}$ is a sequence in $(0,1-k)$ with $\liminf _{n \rightarrow \infty} \beta_{n}>0, k=\max \left\{\tau_{1}, \tau_{2}, \ldots, \tau_{N}\right\} \in(0,1)$. If $\Gamma:=\bigcap_{i=1}^{N} F\left(S_{i}\right) \bigcap_{i=1}^{N} F\left(T_{i}\right) \neq \emptyset$, then the sequence $\left\{x_{n}\right\}$ converges weakly to a point $x^{*} \in \Gamma$, which is a solution of the convex feasibility problem for mappings $\left\{T_{i}\right\}_{i=1}^{N}$ and $\left\{S_{i}\right\}_{i=1}^{N}$.

\subsection{Application to the mixed variational inequality problem of Browder type}

A variational inequality problem (VIP) is formulated as a problem of finding a point $x^{*}$ with property $x^{*} \in C,\left\langle A x^{*}, z-x^{*}\right\rangle \geq 0, \forall z \in C$. We will denote the solution set of VIP by $V I(A, C)$. We know that given a mapping $T: C \rightarrow C$, let $F(x, y)=\langle T x, y-x\rangle$ for all $x, y \in C$. Then $x^{*} \in E P(F)$ if and only if $x^{*} \in C$ is a solution of the variational inequality $\langle T x, y-x\rangle \geq$ 0 for all $y \in C$, i.e., $x^{*}$ is a solution of the variational inequality.

In [20], the mixed variational inequality of Browder type (VI) is shown to be equivalent to finding a point $u \in C$ such that

$$
\langle A u, y-u\rangle+\varphi(y)-\varphi(u) \geq 0, \quad \forall y \in C .
$$


We will denote the solution set of a mixed variational inequality of Browder type by $V I(A, C, \varphi)$

A mapping $A: C \rightarrow H$ is said to be an $\alpha$-inverse-strongly monotone mapping if there exists a constant $\alpha>0$ such that $\langle A x-A y, x-y\rangle \geq \alpha\|A x-A y\|^{2}$ for any $x, y \in C$. Setting $F(x, y)=\langle A x, y-x\rangle$, it is easy to show that $F$ satisfies conditions (A1)-(A4) as $A$ is an $\alpha$ inverse-strongly monotone mapping. Then it follows from Theorem 3.1 that the following result holds.

Theorem 4.3 Let $C$ be a nonempty and closed convex subset of a real Hilbert space $H$, let $A: C \rightarrow H$ be an $\alpha$-inverse-strongly monotone mapping, and let $\varphi: C \rightarrow R \cup\{+\infty\}$ be a proper lower semi-continuous and convex function such that $C \cap \operatorname{dom} \varphi \neq \emptyset$. Let $T_{i}$ : $C \rightarrow C$ be a uniformly $L_{i}$-Lipschitzian and $\tau_{i}$-asymptotically strictly pseudononspreading mapping with the sequence $\left\{k_{n}\right\} \subset[1,+\infty)$ such that $\sum_{n=1}^{\infty}\left(k_{n}-1\right)<\infty$, let $S_{i}: C \rightarrow C$ be a uniformly $\widetilde{L}_{i}$-Lipschitzian and $l_{i}$-asymptotically strictly pseudononspreading mapping with the sequence $\left\{\rho_{n}\right\} \subset[1,+\infty)$ such that $\sum_{n=1}^{\infty}\left(\rho_{n}-1\right)<\infty, i=1,2, \ldots, N$. Let $\left\{x_{n}\right\}$ be a sequence generated by

$$
\left\{\begin{array}{l}
\forall x_{1} \in C, \\
\left\langle A u_{n}, y-u_{n}\right\rangle+\varphi(y)-\varphi\left(u_{n}\right)+\frac{1}{r_{n}}\left\langle y-u_{n}, u_{n}-x_{n}\right\rangle \geq 0, \quad \forall y \in C, \\
y_{n}=\left(1-\beta_{n}\right) u_{n}+\beta_{n} T_{n(\bmod N)}^{n} u_{n}, \\
x_{n+1}=\left(1-\alpha_{n}\right) y_{n}+\alpha_{n} S_{n(\bmod N)}^{n} y_{n},
\end{array}\right.
$$

where $\left\{\alpha_{n}\right\}$ is a sequence in $(0,1)$ with $\liminf _{n \rightarrow \infty} \alpha_{n}>0,\left\{\beta_{n}\right\}$ is a sequence in $(0,1-k)$ with $\liminf _{n \rightarrow \infty} \beta_{n}>0, k=\max \left\{\tau_{1}, \tau_{2}, \ldots, \tau_{N}\right\} \in(0,1)$, and the sequence $\left\{r_{n}\right\} \subset(0, \infty)$ satisfies $\liminf _{n \rightarrow \infty} r_{n}>0$ and $\lim _{n \rightarrow \infty}\left|r_{n+1}-r_{n}\right|=0$. If $\Gamma:=\bigcap_{i=1}^{N} F\left(S_{i}\right) \bigcap_{i=1}^{N} F\left(T_{i}\right) \cap V I(A, C, \varphi) \neq \emptyset$, then the sequence $\left\{x_{n}\right\}$ converges weakly to a point $x^{*} \in \Gamma$.

\section{Competing interests}

The authors declare that they have no competing interests.

\section{Authors' contributions}

Both authors contributed equally to this work. Both authors read and approved the final manuscript.

\section{Author details}

'School of Information Engineering, The College of Arts and Sciences Yunnan Normal, Long quan Road, Kunming, 650222, China. ${ }^{2}$ College of Statistics and Mathematics, Yunnan University of Finance and Economics, Long quan Road, Kunming, 650221, China.

\section{Acknowledgements}

The authors would like to express their thanks to the reviewers and editors for their helpful suggestions and advice. This work was supported by the National Natural Science Foundation of China (Grant No. 11361070).

Received: 19 December 2013 Accepted: 8 April 2014 Published: 06 May 2014

References

1. Blum, E, Oettli, W: From optimization and variational inequalities to equilibrium problems. Math. Stud. 63, 123-145 (1994)

2. Noor, M, Oettli, W: On general nonlinear complementarity problems and quasi-equilibria. Matematiche 49, 313-346 (1994)

3. Jaiboon, C, Kumam, P: A hybrid extragradient viscosity approximation method for solving equilibrium problems and fixed point problems of infinitely many nonexpansive mappings. Fixed Point Theory Appl. 2009, Article ID 374815 (2009)

4. Chang, SS, Chan, CK, Joseph Lee, HW: Modified block iterative algorithm for quasi- $\boldsymbol{\phi}$-asymptotically nonexpansive mappings and equilibrium problem in Banach spaces. Appl. Math. Comput. 217, 7520-7530 (2011)

5. Moudafi, A, Thera, M: Proximal and Dynamical Approaches to Equilibrium Problems. Lecture Notes in Economics and Mathematical Systems, vol. 477, pp. 187-201. Springer, Berlin (1999) 
6. Peng, JW, Yao, JC: A viscosity approximation scheme for system of equilibrium problems, nonexpansive mappings and monotone mappings. Nonlinear Anal. 71,6001-6010 (2009)

7. Chang, SS, Chan, CK, Joseph Lee, HW, Yang, L: A system of mixed equilibrium problems, fixed point problems of strictly pseudo-contractive mappings and nonexpansive semi-groups. Appl. Math. Comput. 216, 51-60 (2010)

8. Kumam, $\mathrm{P}$, Jaiboon, C: A new hybrid iterative method for mixed equilibrium problems and variational inequality problem for relaxed cocoercive mappings with application to optimization problems. Nonlinear Anal. Hybrid Syst. 3(4), 510-530 (2009)

9. Kohsaka, F, Takahashi, W: Fixed point theorems for a class of nonlinear mappings relate to maximal monotone operators in Banach spaces. Arch. Math. 91, 166-177 (2008)

10. Kohsaka, F, Takahashi, W: Existence and approximation of fixed points of firmly nonexpansive-type mappings in Banach spaces. SIAM J. Optim. 19, 824-835 (2008)

11. Iemoto, S, Takahashi, W: Approximating common fixed points of nonexpansive mappings and nonspreading mappings in a Hilbert space. Nonlinear Anal. 71, 2080-2089 (2009)

12. Browder, FE, Petryshyn, WV: Construction of fixed points of nonlinear mappings in Hilbert spaces. J. Math. Anal. Appl. 20, 197-228 (1967)

13. Osilike, MO, Isiogugu, FO: Weak and strong convergence theorems for nonspreading type mappings in Hilbert spaces. Nonlinear Anal. 74, 1814-1822 (2011)

14. Kurokawa, Y, Takahashi, W: Weak and strong convergence theorems for nonspreading mappings in Hilbert spaces. Nonlinear Anal. 73, 1562-1568 (2010)

15. Chang, SS, Cho, YJ, Kim, JK, Zhang, WB, Yang, L: Multiple-set split feasibility problems for asymptotically strict pseudocontractions. Abstr. Appl. Anal. 2012, Article ID 491760 (2012). doi:10.1155/2012/491760

16. Zhao, YH, Chang, SS: Weak and strong convergence theorems for strictly pseudononspreading mappings and equilibrium problem in Hilbert spaces. Abstr. Appl. Anal. 2013, Article ID 169206 (2013). doi:10.1155/2013/169206

17. Quan, J, Chang, SS, Zhang, X: Multiple-set split feasibility problems for $k$-strictly pseudononspreading mapping in Hilbert spaces. Abstr. Appl. Anal. 2013, Article ID 342545 (2013). doi:10.1155/2013/342545

18. Peng, JW, Liou, YC, Yao, JC: An iterative algorithm combining viscosity method with parallel method for a generalized equilibrium problem and strict pseudocontractions. Fixed Point Theory Appl. 2009, Article ID 794178 (2009). doi:10.1155/2009/794178

19. Tan, KK, Xu, HK: Approximating fixed points of nonexpansive mappings by the Ishikawa iteration process. J. Math Anal. Appl. 178, 301-308 (1993)

20. Alber, Yl: Metric and generalized projection operators in Banach space: properties and application. In: Kartosator, AG (ed.) Theory and Applications of Nonlinear Operators of Accretive and Monotone Type, pp. 15-50. Dekker, New York (1996)

10.1186/1687-1812-2014-104

Cite this article as: Ma and Wang: Demiclosed principle and convergence theorems for asymptotically strictly pseudononspreading mappings and mixed equilibrium problems. Fixed Point Theory and Applications 2014, 2014:104

\section{Submit your manuscript to a SpringerOpen ${ }^{\circ}$ journal and benefit from:}

- Convenient online submission

- Rigorous peer review

- Immediate publication on acceptance

- Open access: articles freely available online

- High visibility within the field

- Retaining the copyright to your article 